TM-1465

5501.000

\title{
Flux Jumps in a Pinch
}

\author{
M. Kuchnir \\ Fermi National Accelerator Laboratory \\ P.O. Box 500, Batavia, Illinois 60510
}

July 1,1987 


\section{FLUX JUMPS IN A PINCH}

M. Kuchnir

July 1, 1987

Some clear questions in electromagnetism involving conductors and superconductors do not have obvious answers: How does an electric current distribute itself in a superconductor and in a normal metal? Why is there no plasma pinch effect? What is a flux jump and why does it require the use of multifilamentary composite wires? To answer them we have to involve the foundations of solid state physics.

The pinch effect results from the interaction of free charged particles with the electromagnetic field. In a conductor or superconductor we have no free charged particles but many body constructs called "quasi-particles" that have "dispersion relations", expressions for energy as function of momentum, and obey statistical mechanics as well as scattering laws. Three quasi-particles are relevant to answer these questions: electrons (with mass different than their namesake leptons), phonons and Cooper-pairs.

In a normal metal at room temperature, the atoms form a positively charged lattice and their outermost electrons a highly degenerate gas of free fermions pervading it. The Fermi energy level $\sim 1 \mathrm{eV}$ is much higher than the thermal energy available $(\sim 0.025 \mathrm{eV})$ so very few of these quasiparticles actually participate in the electric current going through the normal metal. Their scattering by impurities, lattice defects and lattice vibrations (phonons) explains the temperature dependence of the resistivity of a metal. This was the phenomenon that Kamerlingh Onnes was studying when he discovered superconductivity.

How does the pinch effect look in this picture? The forces on the outermost leptons due to the current flowing through the metal are small compared to the forces they experience in their interatomic environment. They already are in a pinch! The effect of external magnetic field on the electrons (quasi-particles, not leptons) shows up as an increase in the resistivity of the metal (magneto resistive effect).

The slightly positive wake left in the lattice by an electron passing by can attract another electron. This phonon mediated attraction gives origin to a quasi-particle (called Cooper-pair in the BCS theory) that obeys Bose-Einstein statistics. 
In a superconductor the main charge carriers are bosons with charge -2e. In the condensed state all $N$ of them have the same wave function $\Psi=N$. The electric current density, $\mathrm{J}$, is then given by

$$
J=\frac{h}{2 m i} \quad\left(\Psi^{*} \vec{\nabla} \Psi-\Psi \Psi^{*} \Psi^{*}\right)
$$

and so goes the Landau-Ginzburg formalism describing it. $\Psi \Psi^{*}$ is zero in a magnetic field. In a type I superconductor (like $\mathrm{Pb}, \mathrm{Hg}$, etc), $\Psi^{*}$ is positive over the singly connected volume of the material and no magnetic field exists inside the superconductor, the current flows on the surface and, if the field created by this current goes over $H_{(}(T)$ the material becomes normal. In a type II superconductor (like $\mathrm{NbTi}, \mathrm{Nb}_{3} \mathrm{Sn}$, and apparently $\left.\mathrm{Y} \mathrm{Ba}_{2} \mathrm{Cu}_{3} \mathrm{O}_{7-\delta}\right)$ the magnetic field can penetrate the volume of the material by forming paths of normal zones in this volume. $\Psi^{*}$ is positive over a multiply connected volume inside the material. Inside this multiply connected superconducting volume the magnetic field remains zero, and surface currents surround the magnetic flux lines. Instead of looking at or $\mathrm{J}$ we look at the magnetic field lines. If the distribution of flux lines is uniform the net effect of these surface currents is zero transport current. The existence of a transport current corresponds to gradients in the density of flux lines as the drawing ${ }^{1}$ shows.
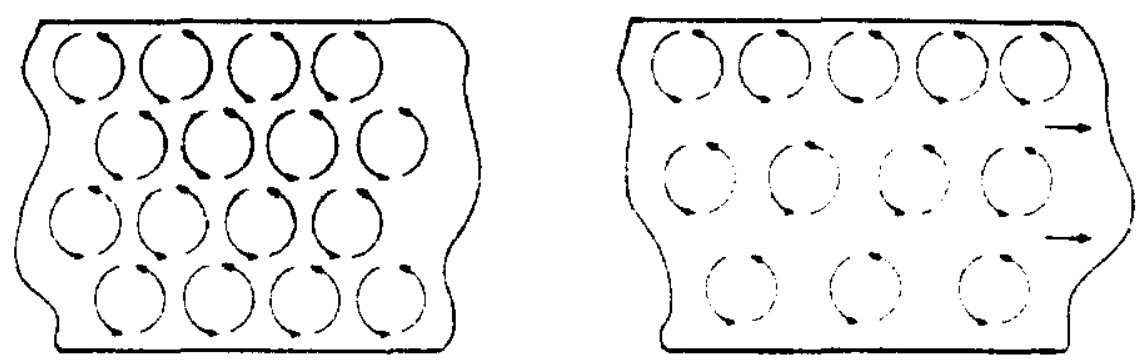

If there is no material defects pinning the flux lines and permitting these gradients to exist, a transport current in a given direction would cause a diffusion of flux lines in the plane perpendicular to that direction. This motion of flux lines dissipates energy and causes the material to warm up, hopefully not enough to make it normal but in any case there is energy dissipation.

The NbTi used in superconducting cables, undergoes a cold work and heat treatment process that creates a large and uniform concentration of defects that serve as strong pinning sites for magnetic flux lines. These pinning sites permit a rather strong gradient of flux line density to get established when magnetic fields and transport currents are imposed. If one of these pinning sites lets its flux line go, it starts a positive feedback event known as "flux jump". The energy released locally by the flux line getting loose raises the local temperature and that reduces the effectiveness 
of neighboring pinning sites besides the value of the critical current density locally which causes flux rearrangement, heat and more pinning sites to let their flux lines go and so on. The heat generated by such an event can be conducted away before the material goes normal if the superconductor has large enough surface area hence the filamentary nature of the $\mathrm{NbTi}$ in the superconducting cables.

How does the pinch effect look in this picture? The superconductor described is the picture of the pinch itself to which magnetic fields cause the bizarre effects described above and to which we got used.

\section{REFERENCE}

1. Martin N. Wilson "Superconducting Magnets" Clarendon Press Publications (1983) p. 284. 